\title{
Breakdown limit studies in high-rate gaseous detectors
}

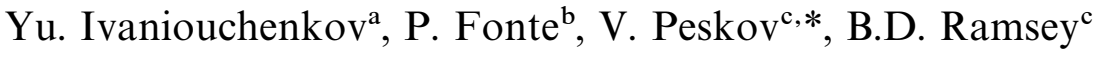 \\ ${ }^{a}$ LIP, Coimbra University, Portugal \\ ${ }^{\mathrm{b}}$ LIP, Coimbra University and ISEC, Coimbra, Portugal \\ ${ }^{\mathrm{c}} N A S A /$ Marshall Space Flight Center, ES-84, Huntsville, AL 35812, USA
}

\begin{abstract}
We report results from a systematic study of breakdown limits for novel high-rate gaseous detectors: MICROMEGAS, CAT and GEM, together with more conventional devices such as thin-gap parallel-mesh chambers and high-rate wire chambers. It was found that for all these detectors, the maximum achievable gain, before breakdown appears, drops dramatically with incident flux, and is sometimes inversely proportional to it. Further, in the presence of alpha particles, typical of the breakgrounds in high-energy experiments, additional gain drops of 1-2 orders of magnitude were observed for many detectors. It was found that breakdowns at high rates occur through what we have termed an "accumulative" mechanism, which does not seem to have been previously reported in the literature. Results of these studies may help in choosing the optimum detector for given experimental conditions. (C) 1999 Elsevier Science B.V. All rights reserved.
\end{abstract}

Keywords: High-rate gaseous detectors; MICROMEGAS; CAT; GEM; Breakdown limits

\section{Introduction}

Future high-luminosity experiments make serious demands on detector technologies and have prompted a chain of inventions of new high-rate gaseous detectors: MicroStrip Gas Counters (MSGCs) [1], MicroGap Counters (MGCs) [2], CAT [3], MICROMEGAS [4] and the Gas Electron Multiplier (GEM) [5]. Due to the extremely tight timescales involved, some of these detectors

\footnotetext{
* Corresponding author. Tel.: + $12565447745 / 9345$; fax: +1256544 7754; e-mail: peskovd@sslob.msfc.nasa.gov
}

were almost immediately adopted for the large experiments at CERN and elsewhere. The aim of our work is to perform an independent systematic study of the breakdown limits of these and other gaseous detectors recently chosen or considered as candidates for high-luminosity applications. Earlier in this study we investigated the breakdown limits of MSGCs, MGCs and both MSGCs and MGCs combined with various preamplification structures [6-8]. Here, we extend our study to incorporate novel high-rate gaseous detectors that have recently appeared in the literature: MICROMEGAS, CAT and GEM, and compare these to more conventional devices such as thin-gap parallel-mesh chambers and high-rate wire chambers. 


\section{Experimental setup}

The experimental setup is described in detail in Refs. $[6,8]$. It consists, in essence, of a test chamber, with a variable absorption and drift region (3-35 mm), inside of which the various detectors under study were installed. We investigated the rate behavior of the following detectors: 3-mm-gap PPACs, 0.6-mm-gap (thin gap) PPACs [9], CAT, GEM, MICROMEGAS, and standard and thickanode MWPCs. The detailed descriptions of most of these designs are given in Refs. [6-8]. In these tests we also, in addition to that described in the above references, used another GEM with hole diameters of $75 \mu \mathrm{m}$ and a hole pitch of $140 \mu \mathrm{m}$. To avoid problems of charge extraction from this GEM its anode was placed in direct contact with a metallic plate (as was suggested in Ref. [10]).

The standard-anode MWPC tested had $20 \mu \mathrm{m}$ anodes on a $3 \mathrm{~mm}$ pitch with a $4 \mathrm{~mm}$ gap between cathode planes, the lower of which was a solid metallic sheet and the upper was an $80 \%$ transparent mesh with a drift space above. The thick-anode MWPC had anode wires of diameter $0.75 \mathrm{~mm}$ on a $6 \mathrm{~mm}$ pitch with an anode-cathode distance of $6 \mathrm{~mm}$. The geometry of this detector lies between that of a PPAC and a conventional wire chamber and was designed to offer high-rates capabilities together with self-quenched streamer discharges [11]. Finally, additional studies were done combining the devices listed above with a subset of these: GEM, PPAC or MICROMEGAS, operated as preamplification structures [6-8].

Most of the measurements were performed in Ar-based mixtures at a pressure of $1 \mathrm{~atm}$, although other mixtures and pressures were occasionally tested [6]. As sources of primary ionization, we used an X-ray gun with variable photon energies $(6,17$ or $30 \mathrm{keV}$ ), and alpha particles collimated perpendicular to the detector surfaces. The full procedures for gain calibration and the measurements of breakdown limits are described in Refs. [6,9].

\section{Results}

The principal results of our studies can be summarized as follows. At low counting rates the breakdown in the thin-gap PPAC, MICROMEGAS and the thick-wire MWPC occurs at some device- and gas-mixture-specific total charge in the avalanche, $\sim 3 \times 10^{7}-10^{8}$ electrons. We assume that the same is true for CAT and GEM (see below). This observation is derived from our measurements with alphas where, by varying the drift gap, we were able to introduce different total charges into the detectors. Breakdown always occurred at some approximately fixed total charge in the avalanche, $\sim 2-6 \times 10^{7}$ electrons. Similar observations were noted for GEM in Ref. [12]. In the case of X-rays, however, both GEM and CAT experienced charge breakdown at lower gains than expected from the total charge rule. This is probably due to surface discharges between the anode and cathode surfaces which may appear at the elevated voltages necessary to detect low primary charge breakdown.

Figs. 1 and 2 depict the measured rate dependence of the maximum achievable gain before breakdown appears. In Fig. 1 we plot the maximum

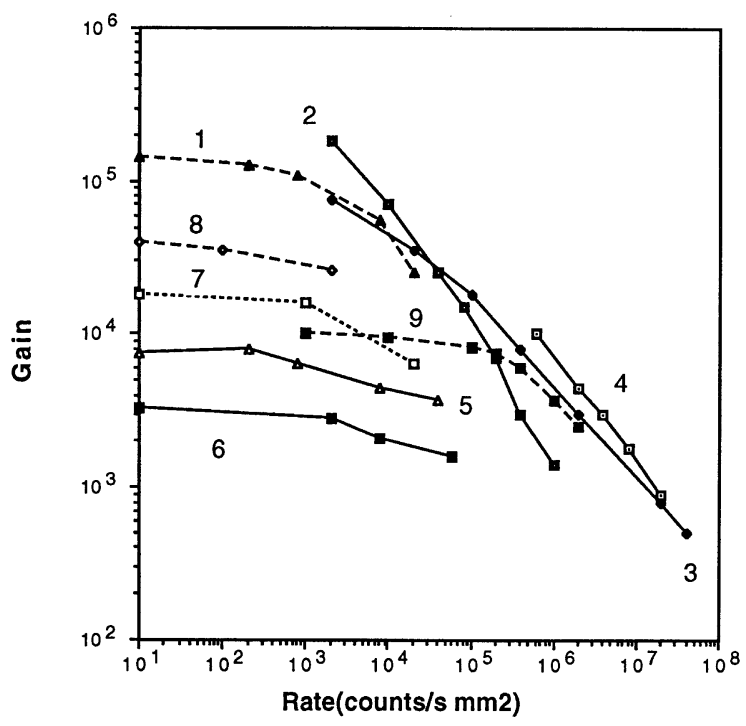

Fig. 1. The maximum achievable gain (curves 1-6), as a function of X-ray flux for various detectors: (1) thick-wire MWPC, (2) PPAC with $3 \mathrm{~mm}$ gap, (3) PPAC with $0.6 \mathrm{~mm}$ gap, (4) MICROMEGAS (from Ref. [13]), (5) CAT, (6) GEM. (7-9) Spacecharge gain limit as a function of rate for other MWPCs: (7) "standard" MWPC, (8) MWPC replotted (from Ref. [14]), (9) thin-gap MWPC (from Ref. [15]). 


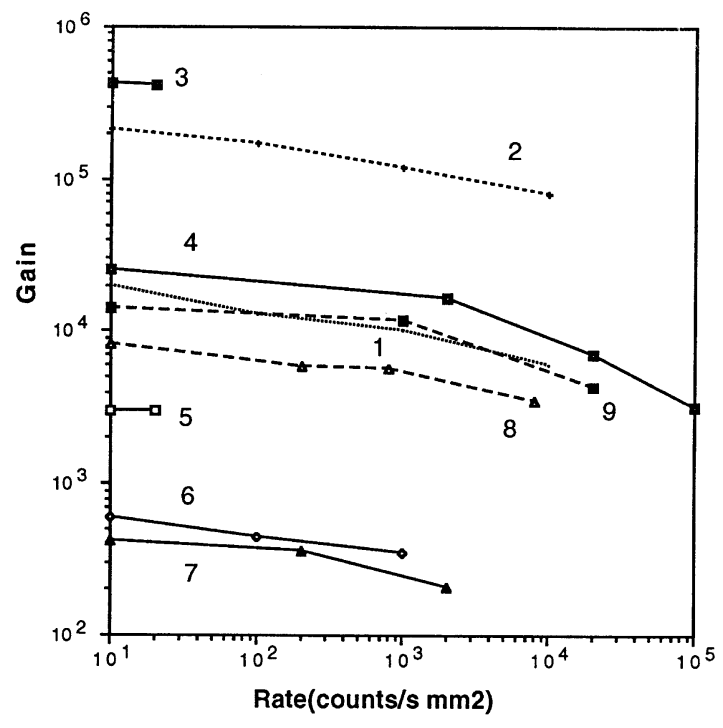

Fig. 2. Maximum achievable gain (curves 1-8) as a function of $\mathrm{X}$-ray flux in the presence of alphas for: (1) diamond-coated MSGC with $0.2 \mathrm{~mm}$ pitch (from Ref. [16]), (2) MSGC (1) with GEM, (3) MSGC, $1 \mathrm{~mm}$ pitch, with PPAC preamplification, (4) PPAC with $3 \mathrm{~mm}$ gap, (5) PPAC with $0.6 \mathrm{~mm}$ gap, (6) CAT, (7) GEM, (8) thick-wire MWPC, (9) space-charge-limited gain variation for the "standard" MWPC in the presence of alphas. The drift space above the detectors were: for (1-4) $3 \mathrm{~mm}$, for (5) $8 \mathrm{~mm}$, for $(6,7) 30 \mathrm{~mm}$ and for $(8,9) 6 \mathrm{~mm}$.

achievable gain before breakdown appears under irradiation with $6 \mathrm{keV}$ X-rays. As one can clearly see, for all the detectors tested the maximum achievable gain drops with rate. We should note that, in the case of the measurements with MICROMEGAS, we observed some charging effects at high rates. The maximum achievable gain corrected for this charging dropped much more rapidly with rate than the raw data would imply. To avoid any possible mistake in the interpretation and correction of these data we used data from Ref. [13] in Fig. 1. One can see from this figure that the highest-rate gains were obtained with MICROMEGAS, the thin-gap PPAC [9] and the thickwire MWPC.

It is interesting to note that the use of any preamplification structure for MICROMEGAS, or the thin-gap or regular PPAC, did not give any significant increase of the total gain [6,9]. A possible explanation of this effect is given in Ref. [8].
During these studies we discovered an interesting feature of the MICROMEGAS and thin-gap PPAC detectors: the discharge which appears at breakdown is self-quenched. That is, the energy released in the spark is much less than expected from the accumulated detector capacity [9]. This feature makes them unique for many applications.

One should note that under X-ray irradiation only, the absolute value of the maximum achievable gain before breakdown occurs is gas-mixture dependent and this may give an extra parameter for detector optimization. However, in the presence of alphas, the maximum total charge was essentially independent of gas mixture and in all cases the maximum achievable gain dropped with rate.

For comparison Fig. 1 also shows data for the MWPCs above and for thin-gap MWPCs taken from Refs. $[14,15]$. In these cases, the high counting rate does not trigger any breakdowns, but lowers the amplitudes due to an accumulation of space charge. Fig. 1, therefore, shows not the maximum gain, but the changes in amplitudes due to the space-charge effect. It is perhaps surprising to see that these detectors have reasonable rate capabilities too.

In Fig. 2 we present results under identical Xray-irradiation conditions to those in Fig. 1, but with the addition of a low rate component of alpha particles. In the case of the "PPAC"-type detectors (PPAC, thin-gap PPAC, GEM and CAT), an additional drop of 1-2 orders of magnitude (depending on the energy deposit in the drift gap) was observed. In this environment the highest gains were achieved with the MSGC combined with a preamplification structure [6].

\section{Discussion}

It is well known that breakdowns in PPACs appear at a critical total charge in the avalanche [17] and the same was recently found to hold true for MICROMEGAS and thin-gap PPACs $[18,19]$. Since CAT and GEM have field configurations similar to MICROMEGAS, one can expect a similar behavior for these devices. In practice, however, this effect can be masked by surface-type breakdowns on the dielectric separating the two 
electrodes since the voltage required to detect low primary ionization is much higher [7].

On closer inspection, the fact that small-gap detectors break down at some total charge in the avalanche is rather surprising as the positive ions are removed from the discharge gaps so fast that a significant accumulation of charge is impossible. Under these conditions the use of the classical Raether limit [17] is perhaps inappropriate. To attempt to explain breakdown limits under these circumstances, one can introduce the concept of a limiting current density which each detector can accept before breakdown. This concept was checked experimentally for parallel-plate chambers in Ref. [19].

The observation that the maximum achievable gain in ALL the gaseous detectors tested drops dramatically, in some cases inversely proportional, with count rate (so that the current density remains constant) and that in the presence of alpha particles, typical of the backgrounds in high-energy experiments, additional gain drops of 1-2 orders of magnitudes may appear, permit clear recommendations for choosing the optimum detector for particular experimental conditions. For example, for measurements of strong $\mathrm{X}$-ray radiation (such as synchrotron radiation), MSGC + GEM [6,8], MICROMEGAS, and thin-gap PPACs will all be ideal candidates. However in the presence of alphas, a good choice would be the MSGC with a preamplification structure or even a conventional or thin-gap MWPC if their spatial resolution can satisfy the user's requirements. Of course, MICROMEGAS, CAT and thin-gap PPACs still can operate at relatively high gains in the presence of alphas, but in this case the drift space should be reduced to $1 \mathrm{~mm}$ or less to severely limit the incident primary charge.

It is interesting to note that rate-limiting breakdowns in most high-rate detectors do not occur through any of the three standard breakdown mechanisms invoked at low rates (streamers or two types of feedback loops) $[6,17]$. For example, in the "PPAC"-type detectors (PPAC, MICROMEGAS, thin-gap PPAC, CAT and GEM) high-incidentflux breakdowns occur through a memory effect: that is, the discharge gap somehow remembers the previous avalanche (for time intervals often much longer than the removal time of the ions!) and this lowers the breakdown limit. We call this new breakdown mechanism "accumulative" breakdown. The results of a detailed study of this type of breakdown are given in Ref. [20].

\section{Conclusion}

We found that in all the detectors tested, breakdowns in the presence of alphas appeared at some critical total charge in the avalanche. From this point of view all these detectors belong to the "PPAC" family.

The highest achievable gains for $\mathrm{X}$-rays alone (and presumably for minimum ionizing particles) were obtained in preamplified-MSGC's MICROMEGAS and thin-gap PPACs. However, in the presence of heavily ionizing particles, both the MICROMEGAS and thin-gap PPACs suffer in performance, and the MSGC + PPAC or the MSGC + GEM combinations offer the highest gains due to diffusion effects [8]. In some high-rate measurements, the standard or thin-gap MWPC can also be used if its spatial resolution satisfies requirements.

\section{Acknowledgements}

We thank G. Charpak, F. Sauli, A. Policarpo, L. Ropelewski and Y. Giomataris for useful discussions.

\section{References}

[1] A. Oed, Nucl. Instr. and Meth. A 263 (1988) 351.

[2] F. Angelini et al., Nucl. Instr. and Meth. A 335 (1993) 69.

[3] F. Bartol et al., J. Phys. III France 6 (1996) 337.

[4] Y. Giomataris et al., Nucl. Instr. and Meth. A 376 (1996) 29.

[5] F. Sauli, Nucl. Instr. and Meth. A 386 (1997) 531.

[6] P. Fonte et al., Nucl. Instr. and Meth. A 419 (1998) 405.

[7] V. Peskov et al., IEEE Trans. Nucl. Sci. 45 (1998) 244.

[8] P. Fonte et al., A study of breakdown limits in microstrip gas counters with preamplification structures, to be published in Nucl. Instr. and Meth. (1998).

[9] P. Fonte et al., Thin gap parallel-mesh chamber: a sparkless high-rate detector, Preprint, LIP 97-05, 1997.

[10] L. Ropelewski, private communication. 
[11] P. Fonte et al., Streamers in MSGC's and other gaseous detectors, ICFA Instr. Bull, Spring, 1997.

[12] F. Sauli, private communication.

[13] Y. Giomataris, private communication, March 1998.

[14] A.H. Walenta, Phys. Scripta 23 (1981) 354.

[15] R.A. Levis et al., Nucl. Instr. and Meth. A 392 (1997) 42.

[16] R. Bouclier et al., Nucl. Instr. and Meth. A 396 (1997) 50.

[17] H. Raether, Electron avalanches and breakdowns in gases, Butterworth, Washington, 1964. P. Fonte et al., Nucl. Instr. and Meth. A 305 (1991) 91.
[18] B. Ramsey et al., Instrumentation for X-ray astronomy from high-altitude balloons: recent developments and future plans, Preprint, NASA, 1997.

[19] P. Fonte et al., Breakdown studies of PPAC's, Preprint, LIP 97-07, 1997.

[20] P. Fonte et al., Fundamental gain limitations of gaseous detectors and optimum designs for high-rate applications, Report submitted to the IEEE-98 Nucl. Sci. Symp., 1998. 